\title{
A Study on Comparison and Evaluation of Sensitivity and Specificity of Different Staining Methods with Conventional Polymerase Chain Reaction among Meningitis Cases - A Prospective Cross Sectional Study
}

\author{
Sunitha Pakalapaty $^{1 *}$, I. Jahnavi ${ }^{1}$, K. Nagamani1 ${ }^{1}$, K. Prashanthi ${ }^{2}$, Manisha Rani ${ }^{1}$, \\ K. Naga Soujanya ${ }^{1}$, G. Sushma Rajya Lakshmi ${ }^{1}$ and Swathi Akula ${ }^{3}$ \\ ${ }^{1}$ Department of Microbiology, Gandhi Medical College, Secundrabad, Telangana, India \\ ${ }^{2}$ Department of Microbiology, Guntur Medical College, Andhra Pradesh, India \\ ${ }^{3}$ Department of Microbiology, Kamineni Institute of Medical Science, L.B. Nagar, Telangana, India \\ *Corresponding author
}

\section{A B S T R A C T}

\section{Keywords}

Mycobacterium, Ziehl Neelsen staining, Flourscent, Polymerase Chain reaction, Tuberculosis.

\section{Article Info}

Accepted: 04 October 2017 Available Online: 10 December 2017
A fast and accurate diagnosis is necessary to control and eliminate tuberculosis (TB). In India TB continues to be a serious public health problem. In this study, diagnostic tests on clinical samples from patients suspected to have TBM were performed and the sensitivity and specificity of the various techniques were compared. The main objective of the study was to compare various staining methods such as acid fast staining and fluorescent staining with conventional polymerase chain reaction and evaluate their sensitivity and specificity for detecting TBM. We have developed specific molecular method suitable for detection of mycobacterium tuberculosis bacteria in CSF of TBM patients. The present study was conducted over a period of one and a half year from March 2014 - August 2015, 100 CSF samples collected from clinically suspected cases of TBM from various wards and ICUs of Gandhi Hospital were tested with smear microscopy, acid fast staining, fluorescent staining and conventional polymerase chain reaction by using different molecular probes. The study results revealed that among 100 clinically suspected cases, 12 cases were confirmed TBM by PCR. The sensitivity and specificity of Ziehl Neelsen staining was $8.3 \%$ and $100 \%$ respectively. Positive predictive value (PPV) and Negative Predictive Value (NPV) of Ziehl Neelsen staining was $100 \%$ and $88.88 \%$ respectively when compared to PCR. One sample which was positive by Ziehl Neelsen staining was also positive by PCR. In addition to this, PCR could pick up 11 more cases which were negative by Ziehl Neelsen staining. Sensitivity and specificity of fluorescent staining was $16.6 \%$ and $100 \%$ respectively when compared to PCR. Positive predictive value and negative predictive values of fluorescent staining was $100 \%$ and $89.7 \%$ respectively when compared to PCR. Two samples that were positive by Fluorescent staining were also positive by PCR. In addition to this PCR could pick up 10 mores cases which were negative by Fluorescent staining. The $\mathrm{p}$ value was found to be significant statistically which is $<0.05$. Among 100 clinically suspected cases of TBM, multiplex PCR was able to detect 12 cases where as uniplex PCR detected 2 cases. The study results can be used to improve existing TB diagnosis procedure as well as for comparing the effectiveness of the Polymerase chain reaction with staining methods. TBM is still a diagnostic problem, so we need accurate detection for diagnosis and treatment. PCR is simple, sensitive, reliable and most effective molecular method for detection of TBM in CSF of Tuberculosis patients. 


\section{Introduction}

Tuberculosis has for centuries continued to be a public health problem of enormous importance, particularly in the developing world. In India, about 22 lakh cases of tuberculosis are added every year. The mortality rate is $5,00,000$ per year, more than 1,000 per day, i.e., one every minute.

Tuberculous meningitis (TBM) is the most severe form of tuberculosis and causes substantial morbidity and mortality (Marais S, et al., 2010). The early diagnosis of and prompt initiation of chemotherapy for TBM are crucial to a successful outcome. However, the early and accurate detection of Mycobacterium tuberculosis in the cerebrospinal fluid (CSF) of TBM patients still remains a challenge for clinicians, mainly due to the lack of rapid, efficient, and practical detection methods (Thwaites and Tran, 2005).

Currently, mycobacterial culture is the gold standard for detecting M. tuberculosis, but it is time-consuming and requires specialized safety procedures in laboratories (Mishra et al., 2005; Radha Krishna et al., 1990). Serological methods are convenient but lack sensitivity and specificity (Attallah et al., 2003; El-Masry et al., 2008). Although the PCR technique is rapid, it is costly for routine use in developing countries where most tuberculosis cases occur (Dora et al., 2008; Lorino et al., 1999; Pai et al., 2003; Pal and Desai, 2007). Conventional smear microscopy with the Ziehl-Neelsen (ZN) stain is a rapid and practical method for detecting acid-fast bacilli (AFB), especially in low-income countries, due to its rapidity, low cost, and high positive predictive value for tuberculosis (Koch and Cote, 1965). However, the ZiehlNeelsen method is severely handicapped by its low detection rate, ranging from 0 to $20 \%$ for CSF specimens (Trusov et al., 2009;
Yeager et al., 1967) one of the main reasons behind this is that $M$. tuberculosis can hardly be stained by acid-fast dyes once it enters the cells. Another important reason is that the Ziehl-Neelsen method requires a large volume of CSF for TBM diagnosis, as it is incapable of detecting bacilli that are fewer than 10,000 in number per slide or per $\mathrm{ml}$ of specimen (Trusov et al., 2009; Yeager et al., 1967). Therefore, it is important to develop an alternative, cost-effective method for detecting intracellular $M$. tuberculosis. Additionally, knowing which cell type is infected by $M$. tuberculosis in the CSF of TBM patients could help us to unravel new antituberculotic candidates (Gaspar et al., 2008). Methods for the diagnosis of tuberculosis improved in recent years and several molecular techniques for its diagnosis have been introduced for clinical use. Molecular methods provide several advantages, including confirmation of the presence of $M$. tuberculosis within 1 to 3 day (lau et al., 1990; Mishra et al., 2005). Newer molecular techniques such as polymerase chain reaction (PCR), although rapid, are costly to be routinely used in developing countries where most TB cases occur (Goel et al., 1990). To overcome these limitations with conventional methods, quicker, specific, and sensitive diagnostic methods are being sought for the diagnosis of TBM. Many gene amplification techniques for direct detection of $M$. tuberculosis are being attempted in research laboratories, with polymerase chain reaction (PCR) being one of them. Various species-specific primers are available for the detection of $M$. tuberculosis. The present study was conducted to find out the role of PCR using the primers (IS6110, MPB64, Protein B, and TRC4) in the diagnosis of TBM. Also there is no previous literature related to the comparison of Acid fast staining (AFB) smears and Fluorescent staining with conventional chain polymerase reaction. PCR using different primers had significant 
advantage over the conventional staining methods to detect the presence of $M$. tuberculosis in specimens of clinically suspected cases of TBM.

\section{Study centre}

This study was carried out in the Department of Microbiology, Gandhi Medical College, Secunderabad, Telangana during the period March 2014 -August 2015. Ethical clearance was obtained from institutional ethical committee. Informed or written consent was taken from the patients or relatives and clinical and demographic data was obtained by structured proforma.

\section{Materials and Methods}

\section{Collection of samples}

100 CSF samples collected from clinically suspected cases of TBM from various wards and ICUs of Gandhi Hospital were processed.

Selection of cases was according to Thwaites et al., [124] clinical entry criteria for Tuberculosis meningitis diagnosis which is defined as symptoms and signs of meningitis including one or more of the following:

Headache, meningeal irritability vomiting, fever, neck stiffness, convulsions, focal neurological deficits, altered consciousness and lethargy.

\section{Study period}

The present study was conducted over a period of one and a half year from March 2014 - August 2015.

\section{Study type}

Prospective Cross - sectional study.

\section{Sample size}

Type of sample- Cerebrospinal fluid (CSF) Sample size for the study was 100 clinically suspected cases of tuberculous meningitis.

\section{Inclusion criteria}

Headache, meningeal irritability, vomiting, fever, neck stiffness, convulsions, focal neurological deficits, altered consciousness and lethargy with any one of the following findings:

Biochemical - for CSF, proteins more than $60 \mathrm{mg} \%$, sugar $<45 \mathrm{mg} \%$ or $<2 / 3^{\text {rd }}$ of blood sugar

Pathological- cell count more than 5-10/hpf, lymphocytic pieocytosis

Radiological -CT scan showing tubercular changes

Supportive evidence - family history of tuberculosis/positive tuberculin test.

HIV reactive cases

All age groups in both the sexes

\section{Exclusion criteria}

All cases which show presence of pus cells and bacteria in Gram staining

Trauma cases

\section{Sample collection}

CSF samples drawn aseptically by the treating physician were collected. One part was processed immediately and another part was stored in $-20^{\circ} \mathrm{C}$ for molecular methods. Gram staining, ZN staining, fluorescent staining, CSF analysis, and culture was done by 
inoculation of a centrifuged CSF sample on chocolate agar and blood agar incubated at $37^{\circ} \mathrm{C}$ to rule out pyogenic meningitis.

\section{Microscopical smears}

For each sample, three smears were made from the centrifuged deposit on new glass slides. Smears were air dried and heat fixed. Gram staining, Ziehl Neelsen staining (ZN) and Fluorescent staining was done for each sample. The staining was done as per the standard protocols (Collee et al., 2008; Cruickshank et al., 1975).

\section{Polymerase Chain Reaction}

The main steps involved in PCR are DNA extraction, Amplification and Analysis of product.

DNA was extracted from the samples using Qiagen DNA Extraction mini kit (QIAGEN GERMANY).

In amplification, two types of PCR are performed Multiplex and Uniplex PCR (Kusum Sharma et al., 2013), depending up on cycling conditions.

\section{Multi targeted PCR (Multiplex PCR)}

Designed to amplify IS6110, MPB64 and Protein antigen $b$ was carried out using six primers in MTB complex with band sizes of 123bp for IS6110, 240bp for MPB64, 419bp for Protein antigen b (Kusum Sharma et al., 2013). PCR Cycle was repeated for 40 cycles.

Uniplex PCR designed to amplify 173bp of TRC4 gene was carried out using two primers, in the genome of M. tuberculosis. Band size is $173 \mathrm{bp}$. PCR cycle was repeated for 35 cycles (Ramya Barani et al., 2012).
Working primers were reconstituted to $20 \mathrm{pmol} / \mathrm{ul}$, by adding $100 \mathrm{ul}$ of stock primers solution to 400ul of DEPC molecular grade water. Each reaction volume was $50 \mu$ of master mix.

\section{Controls}

Positive control: M. tuberculosis, H37Rv strain

Negative control: Reagent control without organisms.

\section{Analysis of products:}

The amplified PCR product was subjected to $2 \%$ agarose gel electrophoresis with ethidium bromide and bands were visualized under UV illuminator.

The bands were seen in Gel doc system at different levels

$\begin{array}{ll}\text { IS6110 } & \text { at } 123 \mathrm{bp} \\ \text { MPB64 } & \text { at 240bp } \\ \text { Protein b } & \text { at } 419 \mathrm{bp} \\ \text { TRC4 } & \text { at } 173 \mathrm{bp}\end{array}$

All the observations were noted and analysed.

\section{Data analysis}

All of the smear slides stained by the conventional or modified method were observed under oil immersion at a magnification of $\times 1,000$. A total of 300 visual fields on each slide were observed, among which AFB-positive fields were counted by three experienced observers independently. All of the data were displayed as means \pm standard errors of the means (SEM) and analyzed using one-way analysis of variance (ANOVA). Differences were considered statistically significant when $P<0.05$. 


\section{Results and Discussion}

In the present study among 100 clinically suspected Tuberculous meningitis (TBM) cases, 12 cases were confirmed TBM cases by PCR (Table 1).

Among 100 clinically suspected TBM cases, $62 \%$ were males and $38 \%$ were females with the male to female ratio of 1.63: 1.Most of the them (33\%) belonged to age group 20-29 yrs. (Table 2)

In Confirmed TBM cases most of them belonged to age group of 20-29 yrs. $58 \%$ are males and $41.6 \%$ are females with male to female ratio of 1.4:1(Table 3 ).

Among 100 clinically suspected TBM cases, positivity for AFB by Ziehl-Neelsen staining was $1 \%$ (Table 4, Figure 4).

Among 100 clinically suspected TBM cases, detection of AFB by Fluorescence Microscopy was 2\% (Table 5).

Sensitivity and specificity of Ziehl Neelsen staining was $8.3 \%$ and $100 \%$ respectively when compared to PCR.

Positive Predictive Value (PPV) and Negative Predictive Value (NPV) of Ziehl Neelsen staining was $100 \%$ and $88.88 \%$ respectively when compared to PCR.

One sample which was positive by Ziehl Neelsen staining was also positive by PCR. In addition to this PCR could pick up 11 more cases which were negative by Ziehl Neelsen staining (Table 6).

Sensitivity and specificity of Fluorescent staining was $16.6 \% \%$ and $100 \%$ respectively when compared to PCR. Positive Predictive Value (PPV) and Negative Predictive Value (NPV) of Fluorescent staining was $100 \%$ and $89.7 \%$ respectively when compared to PCR.
Two samples that were positive by Fluorescent staining were also positive by PCR. In addition to this PCR could pick up 10 mores cases which were negative by Fluorescent staining. The $\mathrm{p}$ value was found to be significant statistically which is $<0.05$ (Table 7).

Among 100 clinically suspected cases of TBM, multiplex PCR was able to detect 12 cases where as Uniplex PCR detected 2 cases (Table 8).

Out of the various genes (IS6110, mpb64, protein b, TRC4) screened among clinically suspected cases by multiplex and uniplex PCR, IS611O was detected in all the 12 samples.

Whereas mpb64, protein $\mathrm{b}$ were detected in 7 samples and TRC4 in 2 samples. IS6110 was found to be the sensitive gene which is detected in all the samples (Table 9).

According to Mann-Whitney U - test

The $p$ value was found to be statistically significant $(p<0.05)$

PCR is found to be more sensitive than ADA with significant $p$ value (Table 10).

Tuberculosis meningitis (TBM) is a medical emergency with high mortality and morbidity that requires rapid detection of Mycobacterium tuberculosis for proper management because poor prognosis is directly related to delay in treatment.

To prevent the general increase in frequency and thus decreasing the mortality, new strategies for its control are needed such as multiple diagnostic modalities for rapid detection of $M$. tuberculosis to initiate early ATT, control transmission of TB, which has led to the assessment of several polymerase 
chain reactions (PCR)-based procedures for detection of $M$. tuberculosis DNA in cerebrospinal fluid (CSF) samples (Desai et al., 2002; Brienze, et al., 2001; Deshpande et al., 2007; Cheng et al., 2004: Ho et al., 2013).

Table.1 TBM Positives among clinically suspected cases ( $\mathrm{n}=100)$

\begin{tabular}{|l|c|c|c|}
\hline & TBM positive & TBM negative & Total \\
\hline Cases & $12(12 \%)$ & $88(88 \%)$ & 100 \\
\hline
\end{tabular}

Table.2 Clinically suspected TBM cases $(\mathrm{n}=100)$

\begin{tabular}{|l|c|c|c|}
\hline \multirow{2}{*}{ Age Group } & \multicolumn{3}{|c|}{ Clinically suspected TBM cases $(\mathrm{n}=100)$} \\
\cline { 2 - 4 } & Males (\%) & Females $(\%)$ & Total No $(\mathrm{n}=100)$ \\
\hline $0-9$ Yrs & $7(7 \%)$ & $0(0 \%)$ & 7 \\
\hline $10-19$ Yrs & $3(3 \%)$ & $4(4 \%)$ & 7 \\
\hline $20-29$ Yrs & $19(19 \%)$ & $14(14 \%)$ & 33 \\
\hline $30-39$ Yrs & $11(11 \%)$ & $8(8 \%)$ & 19 \\
\hline $40-49$ Yrs & $6(6 \%)$ & $5(5 \%)$ & 11 \\
\hline $50-59$ Yrs & $11(11 \%)$ & $4(4 \%)$ & 15 \\
\hline$>60$ Yrs & $5(5 \%)$ & $3(3 \%)$ & 8 \\
\hline Total & $62(62 \%)$ & $38(38 \%)$ & 100 \\
\hline
\end{tabular}

Table.3 Demonstration of AFB by Ziehl - Neelsen staining

\begin{tabular}{|l|l|l|}
\hline Sample & No. of cases & No. of Positives \\
\hline CSF & 100 & $1(1 \%)$ \\
\hline Controls & 20 & NIL \\
\hline
\end{tabular}

Table.4 Demonstration of AFB by Auramine phenol staining

\begin{tabular}{|l|l|l|}
\hline Sample & No. of cases & No. of Positives \\
\hline CSF & 100 & $2(2 \%)$ \\
\hline
\end{tabular}

Table.5 Sensitivity of Ziehl Neelsen staining when compared with PCR

\begin{tabular}{|l|l|l|l|}
\hline & PCR positive & PCR negative & Total \\
\hline $\begin{array}{l}\text { Positive by Ziehl Neelsen } \\
\text { staining }\end{array}$ & 01 & 00 & 01 \\
\hline $\begin{array}{l}\text { Negative by Ziehl Neelsen } \\
\text { staining }\end{array}$ & 11 & 88 & 99 \\
\hline Total & 12 & 88 & 100 \\
\hline
\end{tabular}


Table.6 Sensitivity of Fluorescent staining when compared with PCR

\begin{tabular}{|l|l|l|l|}
\hline & PCR positive & PCR negative & Total \\
\hline Positive by Fluorescent staining. & 02 & 00 & $02(2 \%)$ \\
\hline Negative by Fluorescent staining. & 10 & 88 & 98 \\
\hline Total & 12 & 88 & 100 \\
\hline
\end{tabular}

Table.7 Positivity of Multiplex PCR and Uniplex PCR among clinically suspected cases of TBM cases

\begin{tabular}{|l|l|}
\hline MULTIPLEX PCR (IS6110,MPB64,Protein b) & UNIPLEX PCR(TRC4) \\
\hline $12(100 \%)$ & $2(16.6 \%)$ \\
\hline
\end{tabular}

Table.8 Number of samples positive for individual genes among TBM positive cases

\begin{tabular}{|l|l|l|l|}
\hline $\begin{array}{l}\text { Samples positive for } \\
\text { IS6110 }\end{array}$ & $\begin{array}{l}\text { Samples } \\
\text { positive for } \\
\text { MPB64 }\end{array}$ & Samples Positive for & $\begin{array}{l}\text { Samples positive } \\
\text { for IS6110 }\end{array}$ \\
\hline 12 & 7 & 7 & 2 \\
\hline
\end{tabular}

Table.9 Clinical and laboratory parameters of patients with CSF specimen positive by PCR

\begin{tabular}{|l|l|l|l|l|l|l|}
\hline S.No & $\begin{array}{l}\text { Total WBC } \\
\text { count }\end{array}$ & Lymphocytes & Protein & Sugar & Ada & Att outcome \\
\hline 1. & 12 & 96 & 120 & 16 & 8 & Improved \\
\hline 2. & 40 & 80 & 34 & 28 & 12.4 & Improved \\
\hline 3. & 10 & 100 & 146 & 12 & 36 & Improved \\
\hline 4. & 5 & 80 & 144 & 54 & 8 & Improved \\
\hline 5. & 50 & 100 & 388 & 24 & 31 & LAMA \\
\hline 6. & 40 & 70 & 154 & 36 & 7 & Expired \\
\hline 7. & 6 & 100 & 24 & 88 & 8 & Improved \\
\hline 8. & 15 & 80 & 72 & 24 & 12 & Expired \\
\hline 9. & 5 & 95 & 76 & 20 & 10.4 & Improved \\
\hline 10. & 30 & 84 & 148 & 42 & 11.4 & Improved \\
\hline 11. & 6 & 70 & 22 & 56 & 6.1 & Improved \\
\hline 12. & 90 & 80 & 300 & 24 & 13 & Improved \\
\hline
\end{tabular}

This study was undertaken to assess the utility of various diagnostic modalities for early diagnosis of tuberculosis meningitis. Also an attempt was made to compare the sensitivity of PCR with conventional diagnostic techniques for detection of MTB in CSF samples (Cheng et al., 2004: Ho et al., 2013). In the present study out of 100 suspected cases of TBM 12 were positive for Mycobacterium tuberculosis similar to previous studies (Aziz et al., 2004). These results were correlated with other reports, $12.5 \%$ and $13.3 \%$ (Shastry et al., 2013; Hooker et al., 2003). Some other studies also showed low positivity of $1.7 \%$ and $3.7 \%$ respectively (Shirani et al., 2015). Whereas some previous studies reported high positivity of $41.6 \%$, 
$51.7 \%$ and $81.48 \%$ respectively. (Shyam Chand Choudary et al., 2015; Kusum Sharma et al., 2010)

In the present study, male preponderance was observed however it was not significant statistically $(\mathrm{p}=0.78)$.

In the study Male to female ratio among clinically suspected cases of TBM was1.63: 1 . These results were agreement with the findings of other studies 1:63:1 (Caws et al., 2000)' In a male dominated society, usually he is the earning member. As he goes out for work, he is more likely to come in contact with an active Tuberculosis case. Men are more likely to acquire habits like smoking and alcoholism which predispose to TB. In confirmed TBM cases, male preponderance was seen which was also observed (Shirani et al., 2015).

In the present study, most common age group affected among clinically suspected TBM was 20-29 yrs. These results were similar with that of previous findings (Shyam Chand Choudary 2015; Shirani et al., 2015). The reasons that make this age group vulnerable are, they are socially more active and are more exposed to an open case of TB than others. In some parts of the world HIV pandemic has contributed as people of this age group are sexually most active. In confirmed TBM cases, the most common age group affected is $20-29$ yrs. In the present study, Ziehl Neelsen staining could detect $1 \%$ of cases among 100 clinically suspected TBM cases similar to the previous findings (Guyen et al., 1996). Some other findings showed some variation in results, $1.81 \%$, 2.85\% (Kusuma Sharma et al., 2015; Parija et al., 2011).

The detection limit of microscopy is $10^{4}$ mycobacteria per milliliter whereas the majority of patients with TBM have fewer mycobacteria in their CSF samples and are thus missed as tuberculous meningitis cases. In the present study the sensitivity of $\mathrm{ZN}$ staining when compared to PCR is $8.3 \%$ which is nearer to previous findings, $7.72 \%$ sensitivity respectively (Sajjid Iqbal et al., 2011). In some studies conducted and showed higher sensitivity of $40.9 \%$, while some studies reported lower sensitivity of $1.11 \%$ and $1 \%$ respectively (Kusum Sharma et al., 2015; Guyen et al., 1996).

This may be due to paucibacillary nature of specimens. PCR requires nearly 800 organisms/ml whereas Microscopy requires 10,000 bacilli per ml. CSF does contain few bacilli hence the low sensitivity.

In the present study the sensitivity of Auramine phenol staining when compared to PCR is $16.6 \%$ which is nearer to previous reports (Parija et al., 2011), who reported $11.53 \%$ sensitivity respectively. In some studies conducted and reported a lower sensitivity of 4\% (Shastry et al., 2013).

Detection of AFB by Auramine phenol staining requires $10^{4}$ organisms $/ \mathrm{ml}$ like $\mathrm{ZN}$ staining, but Fluorescent microscopy is $10 \%$ more sensitive than $\mathrm{ZN}$ staining and more slides can be screened in lesser time.

Thus, PCR is a powerful and reliable technique for rapid diagnosis of $M$. tuberculosis, with a reported sensitivity of $55-95 \%$ in culture positives but $100 \%$ in both smear- and culturepositive clinical specimens (Lau et al., 1990). The usefulness of PCR in the diagnosis of TB by using a variety of unselected clinical specimens is not clear as studies have differed in techniques including lysing method and target nucleic acid to detect products as well as the number and type of samples used, making the reported sensitivities and specificities difficult to compare (Holodniy et al., 1991; Schluger et al., 1994). A recent study comparing four conventional techniques, FNA cytology, ZN staining, culture and lymph node biopsies, and TB PCR indicated 94.8\% diagnosis but PCR was found to be highly sensitive (94.4\%) though less specific (38.2\%) (Singh et al., 2000; Goel et al., 2001). In the present study, PCR was found to be the most sensitive $(89.5 \%)$ as well as specific 
(86.1\%) method for early diagnosis of tuberculosis.

In the Present study, among 100 clinically suspected cases of TBM, multiplex PCR (MPCR) was able to detect 12 cases where as Uniplex PCR detected 2 cases.

To increase diagnostic accuracy, multiplex PCR has been evaluated using three targets, i.e., IS6110, MPB-64 and protein ' $b$ ' together for diagnosis of Mycobacterium tuberculosis complex. Other studies which have evaluated two or three targets had put up separate PCRs for each reaction, which increased the cost of the test and also the chances of cross contamination. This method of using multiplex PCR for single PCR reaction reduced error, as well as cost, and increased the sensitivity of the test.

Previous studies reported, that multi plex PCR was shown to have good sensitivity and specificity. Multiplex PCR could potentially be used for patient care in endemic resource poor countries, where RT-PCR technologies is not feasible due to their cost and complexity. Multi plex PCR's potentially improved sensitivity and specificity to diagnose TB as well as its easier application in resource poor areas, may hold the potential to give clinicians the tool they need to better diagnose and ultimately manage this deadly disease (Askin et al., 2013).

Previous studies conducted at the Tuberculosis Research Centre have suggested that the sensitivity of PCR can be increased by using two sets of targets (IS6110 and TRC4) to detect tuberculous meningitis and tubercular pleuritis. Some previous studies suggested that the diagnosis of CNS tuberculosis requires not a screening examination but rather a definitive molecular procedure like IS6110 and mpb-64 gene characterization for accurate and confirm diagnosis of tubercular meningitis. It is shown that using multiple targets to detect $\mathrm{TB}$ on a single PCR reaction may increase the sensitivity of NAA tests for the diagnosis of TB (Singh et al., 2015; Sharma et al., 2013).
Out of the various genes (IS6110, mpb64, protein b, TRC4) screened among clinically suspected TBM cases by multiplex and uniplex PCR, 12 were confirmed by PCR, in which IS611O was detected in all the 12 samples, whereas MPB64, protein $b$ were detected in 7 samples and TRC4 in 2 samples. IS6110 had shown $100 \%$ positivity, whereas MPB64 and Protein b had shown a positivity of $58.33 \%$, and TRC4 had shown a positivity of $16.6 \%$.The reason for widely used IS6110 in PCR tests is the presence of its multiple copies in $M$. tuberculosis complex genome, which is believed to confer higher sensitivity.

MPB64 is the most specific sequence for the diagnosis of the MTB complex by PCR, because of lowest number of false positive results obtained (10\%) in comparison to IS6110 $(62 \%)$ and heat shock protein (38\%) The MPB64 gene codes for the MPB64 protein and is a $240 \mathrm{bp}$ region (nucleotides 460-700).

Various studies done and the results were shown that IS6110 is the most sensitive gene, a member of IS3 family found in almost all members of the M. tuberculosis complex and had multiple copies more or less randomly distributed throughout the genome, with copy numbers ranging from 10 to 26 copies, made it an ideal target for PCR (Raffi et al., 2007; Maurry et al., 2012).

The overall aim of this study was to evaluate rapid, sensitive and specific tests like $\mathrm{ZN}$ microscopy, Fluorescent microscopy and PCR for diagnosis of Tuberculosis meningitis. TB PCR was found to be highly sensitive, specific and rapid method for early diagnosis of TBM. Although conventional diagnostic techniques remain the method of choice in regions with low-resource settings, PCR may be employed in cases with strong clinical suspicion and equivocal results, especially at an early stage of the disease, for better diagnosis, management and treatment. Given the acute need for simple, efficient and practical method for detecting $M$. tuberculosis within the cells and from small 
CSF sample. In the present study finally we concluded that, this study shown low sensitivity of Ziehl Neelsen and Fluorescence staining, compared with conventional PCR. Detection of IS6110 PCR was found to be most sensitive, valuable and reliable tool in diagnosing TBM. Therefore, this method will be of tremendous value in improving both the diagnosis and treatment of tuberculosis.

\section{Acknowledgement}

Authors thankful to Department of Microbiology, Gandhi Medical College for providing instrumental facilities (PCR) to carry out this research work.

\section{References}

Ashkin, D., Dean., Fiorella, S.P., Laal, S., Lee, Y., Pedrosa M., K Sharma, K., Sharma, A., Sharma, M., Singh K.K., Singh G and D Willis, D. 2013. Evaluation of multiplex Polymerase chain reaction utilising multiple targets in Mycobacterium tuberculosis direct test negative but culture positive cases: A potential method for enhancing the diagnosis of tuberculosis. Indian Journal of Medical Microbiology., Vol. 31(4): 370-373.

Attallah AM, et al., 2003. Rapid and simple detection of a Mycobacterium tuberculosis circulating antigen in serum using dot-ELISA for field diagnosis of pulmonary tuberculosis. J. Immunoassay Immunochem. 24:73-87.

Aziz, M. M., et al., 2004. Comparison between IS6110 and MPB64 primers for the diagnosis of Mycobacterium tuberculosis in Bangladesh by polymerase chain reaction (PCR). Bangladesh Medical Research Council bulletin., 30(3): 87.

Caws, M., Wilson, S.M., Clough, C and Drobniewski., F.2000. Role of IS6110targeted PCR, culture, biochemical, clinical, and immunological criteria for diagnosis of tuberculous meningitis., $J$. Clin. Microbiol., 38: 3150-3155.
Chaudhary and Shyam Chand, et al., 2015. Evaluation of IS6110 PCR in CSF for the rapid diagnosis of tuberculous meningitis. Annals of Tropical Medicine and Public Health., 8(5): 191.

Cheng, V. C. C., et al., 2004. Clinical evaluation of the polymerase chain reaction for the rapid diagnosis of tuberculosis. Journal of clinical pathology., 57(3): 281-285.

Collee, J.G, Fraser, A.G, Marmion, B.P, Simmons, A., Mackie and McCartney.2008. Practical Medical Microbiology: Staining methods. $14^{\text {th }} \mathrm{ed}$. New Delhi: Reed Elsevier India Private Limited., 793-812.

Cruickshank, R., Duguid, J.P., Marmion, B.P and Swain, R.H.A. 1975. Practical Medical Microbiology. Vol. 2, $12^{\text {th }}$ edn. London: Churchill Livingstone; Pp. 3839.

Desai, M.M and R. B. Pal, R.B. 2002. "Polymerase chain reaction for the rapid diagnosis of tuberculous meningitis. Indian Journal of Medical Sciences., 56(11): 546.

Deshpande, Poonam S., et al., 2007. Evaluation of the IS6110 PCR assay for the rapid diagnosis of tuberculous meningitis. Cerebrospinal Fluid Res., 4(10): 8454.

Dora, J.M, et al., 2008. Polymerase chain reaction as a useful and simple tool for rapid diagnosis of tuberculous meningitis in a Brazilian tertiary care hospital. Braz. J. Infect., Dis. 12:245-247.

El-Masry, S., El-Kady, I., Zaghlou, 1 M.H and Al-Badrawey, M.K. 2008. Rapid and simple detection of a mycobacterium circulating antigen in serum of pulmonary tuberculosis patients by using a monoclonal antibody and Fast-DotELISA. Clin. Biochem.. 41:145-151.

Gaspar, M. M, et al., 2008. Developments on drug delivery systems for the treatment of mycobacterial infections. Curr. Top. Med. Chem., 8:579-591.

Goel, M. M., Ranjan, V., Dhole, T. N., Srivastava, A. N., Mehrotra, A., Kushwaha, M. R. and Jain, A. (2001). 
Polymerase chain reaction vs. conventional diagnosis in fine needle aspirates of tuberculous lymph nodes. Acta Cytol., 45: 333-340.

Guyen, N., Lan N., et al.,1996. The potential contribution of the polymerase chain reaction to the diagnosis of tuberculous meningitis. Archives of neurology., 53(8): 771-776.

Ho, Jennifer, et al., 2013. Diagnosing tuberculous meningitis-have we made any progress?. Tropical Medicine \& International Health., 18(6): 783-793.

Hooker, J. A. G., et al., 2003. Diagnostic utility of cerebrospinal fluid studies in patients with clinically suspected tuberculous meningitis. The International Journal of Tuberculosis and Lung Disease., 7(8): 787-796.

Iqbal, Sajjad, et al., 2011. Importance of polymerase chain reaction in diagnosis of pulmonary and extra-pulmonary tuberculosis. J Ayub Med Coll Abbottabad., 23(1): 1-10.

Koch, M.L and Cote, R.A. 1965. Comparison of fluorescence microscopy with ZiehlNeelsen stain for demonstration of acidfast bacilli in smear preparations and tissue sections. Am. Rev. Respir. Dis., 91:283-284.

Kusum Sharma, Vishali Gupt, Reema Bansal, Aman Sharma, Meera Sharma and Amod Gupta Sharma et al., 2013. Novel multitargeted polymerase chain reaction for diagnosis of presumed tubercular uveitis. Journal of Ophthalmic Inflammation and Infection., 3:25. http://www.joiijournal.com/content $/ 3 / 1 / 25$

Lau, S. K., Wei, W. I., Hsu, C. and Engzell, U. C. (1990). Efficacy of fine needle aspiration cytology in the diagnosis of tuberculous cervicallymphadenopathy. $J$ Laryngol Otol., 104, 24-27.

Lorino, G, et al., 1999. Polymerase chain reaction, with sequencing, as a diagnostic tool in culture-negative bacterial meningitis. Clin. Microbiol. Infect., 5:9296.
Maurya, A.K., Kant, S., Nag, V.L., Kushwaha, R. and Dhole, T.N. 2012. Detection of $123 \mathrm{bp}$ fragment of insertion element IS6110 Mycobacterium tuberculosis for diagnosis of extrapulmonary tuberculosis. Indian journal of medical microbiology., 30(2): 182.

Mishra A, et al., 2005. Direct detection and identification of Mycobacterium tuberculosis and Mycobacterium bovis in bovine samples by a novel nested PCR assay: correlation with conventional techniques. J. Clin. Microbiol., 43:56705678.

Pai M, et al., 2003. Diagnostic accuracy of nucleic acid amplification tests for tuberculous meningitis: a systematic review and meta-analysis. Lancet Infect. Dis. 3:633-643.

Pal, R.B and Desai, M.M. 2007. Polymerase chain reaction for the rapid diagnosis of tuberculous meningitis. J. Indian Med. Assoc., 105:21-24.

Parija, Subhash Chandra, and A. R. Gireesh. 2011. Early diagnosis of tuberculous meningitis: A comparison of nested polymerase chain reaction and BacT/ALERT. Indian Journal of Pathology and Microbiology., 54.2 (2011): 421.

Radhakrishnan, V.V, Sehgal, S and Mathai, A. 1990. Correlation between culture of Mycobacterium tuberculosis and detection of mycobacterial antigens in cerebrospinal fluid of patients with tuberculous meningitis. J. Med. Microbiol., 33:223-226.

Rafi, W., et al., 2007. Role of IS6110 uniplex PCR in the diagnosis of tuberculous meningitis: experience at a tertiary neurocentre. The International Journal of Tuberculosis and Lung Disease., 11(2): 209-214.

Ramya Barani, Gopalsamy Sarangan, Tessa Antony, Soundararajan Periyasamy, Anupma Jyoti Kindo, Padma Srikanth. 2012. Improved detection of Mycobacterium tuberculosis using two independent PCR targets in a tertiary care 
centre in South. IndiaJ Infect Dev Ctries., 6(1):46-52.

rienze, Vânia Maria Sabadoto, et al., 2001. Low sensitivity of polymerase chain reaction for diagnosis of tuberculous meningitis in southeastern Brazil. Revista da Sociedade Brasileira de Medicina Tropical., 34(4): 389-393.

Sastry, Apurba Sankar, and Kumudavathi Sandhya Bhat K. 2013. The diagnostic utility of Bact/ALERT and nested PCR in the diagnosis of tuberculosis meningitis. Journal of clinical and diagnostic research., 7(1): 74.

Schluger, N. W., Kinney, D., Harkin, T. J. and Rom, W. N. 1994. Clinical utility of the polymerase chain reaction in diagnosis of infections due to Mycobacterium tuberculosis. Chest., 105: 1116-1121.

Sharma Kusum, et al., 2010. Evaluation of polymerase chain reaction using protein $b$ primers for rapid diagnosis of tuberculous meningitis. Neurology India, 58(5): 727.

Sharma, K., et al., 2013. Evaluation of multiplex Polymerase chain reaction utilising multiple targets in Mycobacterium tuberculosis direct test negative but culture positive cases: A potential method for enhancing the diagnosis of tuberculosis. Indian journal of medical microbiology., 31(4): 370.

Shirani, Kiana, et al., 2015. Diagnosed tuberculosis meningitis using cerebrospinal fluid polymerase chain reaction in patients hospitalized with the diagnosis of meningitis in referral hospitals in Isfahan. Journal of Research in Medical Sciences., 20(3): 24-19.

Singh, K. K., Muralidharn, M., Kumar, A., Chattopadhyaya, T. K., Kapila, K., Singh, M. K., Sharma, S. K., Jain, N. K. and
Tyagi, J. S. 2000. Comparison of in house polymerase chain reaction with conventional techniques for the detection of Mycobacterium tuberculosis DNA in granulomatous lymphadenopathy. $\mathrm{J}$ Clin Pathol., 53: 355-361.

Singh, S.D., T. Masood, T., Sabharwal, R.K., Sharma, N., Nautiyal, S.C and Singh, R.K. 2015. Biochemical and molecular characterization of cerebrospinal fluid for the early and accurate diagnosis of Mycobacterium tuberculosis. Biomedical Research., 26(3): 426-430.

Thwaites, G. E., Chau, T.T.H., Stepniewska, K., Phu, N. H., Chuong, L.V., Sinh, D.X., White, N.J., Parry, C.M and Farrar, J.J.2002. Diagnosis of adult tuberculous meningitis by use of clinical and laboratory features. Lancet, 360: 12871292.

Thwaites, G.E, and Tran, T.H. 2005. Tuberculous meningitis: many questions, too few answers. Lancet Neurol., 4:160170.

Trusov A, et al., 2009. Comparison of Lumin LED fluorescent attachment, fluorescent microscopy and Ziehl-Neelsen for AFB diagnosis. Int. J. Tuberc. Lung Dis., 13:836-841.

Trusov, A, et al., 2009. Modified immunohistological staining allows detection of Ziehl-Neelsen-negative Mycobacterium tuberculosis organisms and their precise localization in human tissue. J. Pathol., 205:633-640

Yeager, H, Jr, Lacy J, Smith, L.R and LeMaistre, C.A. 1967. Quantitative studies of mycobacterial populations in sputum and saliva. Am. Rev. Respir., Dis. 95:998-1004.

\section{How to cite this article:}

Sunitha Pakalapaty, I. Jahnavi, K. Nagamani, K. Prashanthi, Manisha Rani, K. Naga Soujanya, G. Sushma Rajya Lakshmi and Swathi Akula. 2017. A Study on Comparison and Evaluation of Sensitivity and Specificity of Different Staining Methods with Conventional Polymerase Chain Reaction among Meningitis Cases - A Prospective Cross Sectional Study. Int.J.Curr.Microbiol.App.Sci. 6(12): 157-168. doi: https://doi.org/10.20546/ijcmas.2017.612.021 